\title{
The effectiveness of sleep hygiene program on sleep quality and stress level in elderly population.
}

\author{
Kiran Sharma*, Swati Srivastava \\ Department of Rehabilitation Sciences, Jamia Hamdard, New Delhi, India
}

\begin{abstract}
Background and objective: Elderly are more vulnerable to stress than other age groups due to increase in stressors resulting from declining health and social relationship. Among Geriatric illnesses, geriatric sleep disturbance accounts for as high as $25 \%$ through $60 \%$. The elderly tend to wake up more often during sleeping at night so in order to overcome sleep disorder, not only the drug treatment but also cognitive behavioural programs such as sleep hygiene education can be presented. Hence the study was set out to analyse the effect of sleep hygiene program on sleep quality and stress level in elderly population.

Methodology: A cross-sectional survey design was used. 70 elderly subjects aged over 85 years were taken from community.

They were assessed using following outcome measures:

1. Cohen Perceived Stress Scale

2. Pittsburgh Sleep Quality Index

A Sleep Hygiene Program was developed and its effect was analysed on sleep quality and stress level in the sample.

Result: Within the study Sleep Hygiene Program found to be effective in improving the sleep quality of elderly $(\mathrm{M} \pm \mathrm{SD}=$ pre-1.41 \pm .496 , post-2.64 $\pm \mathbf{0 . 7 4 2}, \mathrm{t}=-\mathbf{9 . 7 7 2}, \mathrm{p}=\mathbf{0 . 0 0 0})$. We identified that maximum number of participants had high stress level $(55 \%)$ and the stress level of the participants was reduced after the implementation of sleep hygiene program.

The findings also revealed that increase stress level was significantly associated with poor sleep quality (Pearson's $r$ values $=$ pre- 0.95 , post-0.92).

Conclusion: From the result we concluded that Sleep Hygiene Program is an important tool for improving the sleep quality and stress level of elderly. There is positive association between sleep and stress.
\end{abstract}

Keywords Sleep hygiene program, Sleep quality, Stress level, Elderly population.

Accepted on March 202018

\section{Introduction}

World Health Organization classified old age as: 65-75 years as young old, 75 to 85 years as old and above 85 years as elderly [1]. Old age is considered to be near about 70 years in developed countries where life expectancy is more due to advancement in health care techniques, as compared to developing countries where the old age is considered near about 60 years because of low life expectancy. Globally, 1\% of population over age of 60 years is increasing and it is likely to reach $30 \%$ of world population by 2050 . According to Census of India (2001), about 7.7\% population is over 60 years of age. This is expected to increase to $17 \%$ in 2050 . As a person chronically ages, an individual gradually or suddenly loses his physical capabilities, physiological resources of body functions, occupations, friends, and spouse and may be independence. Chronic diseases such as diabetes mellitus, coronary heart diseases, osteoporosis and cerebrovascular are most common diseases in elderly people and predisposes them to increased risk to develop stress. Psychosocial stress arises due to problems in area of daily living and sub areas (mobilizing, sleeping, work, play and communication) [2]. Perceived stress occurs when an individual judges that situational demands exceed his or her resources [3]. These situational stressors when become too severe and numerous increases the risk of sleep disorders [4]. Thus, Elderly people have higher probability of suffering from multiple health disorders [5].

Among Geriatric illnesses, geriatric sleep disturbance accounts for as high as $25 \%$ through $60 \%$. Half of elderly individuals report some form of sleep difficulty, which may include longer sleep onset times, lower rates of sleep efficiency, more time in bed, more awakenings during the night, earlier wake up times, and more daytime naps, as they experience changes in bio rhythm to make them sleep early in the evening and wake up early in the morning [6].

Sleep disorder leads to many physical symptoms such as lethargy, fatigueless, lower enthusiasm, heart palpitation, and fever to prolong the sense of stress due to changes in autonomic nervous system. Despite the significant impact of sleep problems reported above, awareness in the community is low. It has also been recognised that sleep education programs could be an effective solution in addressing this deficiency [7]. Therefore, in order to overcome sleep disorder and stress, not 
Citation: Sharma K, Srivastava S. The effectiveness of sleep hygiene program on sleep quality and stress level in elderly population. J Ment Health Aging 2018;2(1):29-32.

only the drug treatment but also cognitive behavioural programs such as sleep hygiene education can be presented. The sleep hygiene program is an easily approachable and an effective intervention method for elderly population, as it encourages environmental improvement and lifestyle change as factors influencing sleep quality and stress level [8].

Poor sleep quality, indicated by subjective sleep ratings, sleeponset times, sleep duration, sleep difficulties, and daytime functioning, can lead to significantly greater psychosocial distress [9]. The little is known about the stress level and sleep disorder faced by the elderly population. The aim of the study is to analyse the effect of sleep hygiene program on sleep quality and stress level in elderly population.

\section{Materials and Methods}

\section{Study design}

A cross-sectional survey was used.

\section{Subjects}

A convenience sample of 70 elderly subjects was taken from community. All male and female elderly participants aged over 85 years and willing to participate were eligible for inclusion. Participants not able to respond to the questions and not willing to participate were excluded.

\section{Procedure}

The purpose of study was explained to all the selected participants in their local language. Verbal consent and written consent were obtained from participants who were selected in the survey. Data was collected by face to face interview method using different questionnaires. Questionnaires were filled by the investigators at the time of interview. Following that all the participants were provided with sleep hygiene program and then its effect was analysed on sleep quality and stress level among the sample.

\section{Sleep hygiene program}

The sleep hygiene program contained 4 sessions over a period of 6 weeks. 1st to 3rd session last for $50 \mathrm{~min}$ and 4th session, which included group session last for $3 \mathrm{~h}$ ? Weekly follow up of participants was done through telephonic interview method. Table shows the sleep hygiene program developed by the researchers in the study.

In Session 1, the basic knowledge about the sleep disorder was provided to the participants. They were introduced about the effect of sleep disorders in their life. Then the introduction about the sleep hygiene was provided to them.

In Session 2, guidelines for better sleep (HOW TO SLEEP BETTER) were provided, and benefits of good sleep were explained to the participants following the general revision of session 1.

In session 3, they were introduced about eat well sleep well program, in which tips for good food for sound sleep was provided.

In Session 4, the effect of sleep disorders on stress was explained in detail, following the revision of all previous sessions. A group session was carried out among all the participants in whom they shared their sleep and stress related problems.

\section{Sleep hygiene booklet}

Participants were given a Sleep hygiene Booklet which covers general information about sleep disorder knowledge, sleep hygiene education, relaxation exercise and quizzes. The booklet was aimed at providing the follow up of sleep hygiene program at home (Table 1).

Table 1. Sleep hygiene booklet.

\begin{tabular}{lll}
\hline No. of Sessions & Topics Discussed & Details \\
\hline 1 & Sleep disorder awareness & $\begin{array}{l}\text { Cause of sleep problems } \\
\text { Effects of sleep disorders } \\
\text { Sleep hygiene: Introduction }\end{array}$ \\
\hline 2 & How to sleep better & $\begin{array}{l}\text { Dos and don'ts for better sleep. } \\
\text { Benefits of good sleep. }\end{array}$ \\
\hline 3 & Eat well sleep well program & Good food for sound sleep \\
\hline 4 & Effect of sleep disorder on stress. & Poor sleep quality can lead to significantly greater psychosocial distress. \\
& Group Discussion. & \\
\hline
\end{tabular}

\section{Outcome measures/scales}

Perceived stress scale (PSS): Perceived Stress Scale is an effective instrument used to assess the degree to which people perceive their lives as stressful. Subjects indicate how often they have found their lives unpredictable, uncontrollable, and overloaded in the last month. It has 10 items. Each item is rated on a 5-point scale ranging from never (0) to almost always (4).
Positively worded items are reverse scored, and the ratings are summed, with higher scores indicating more perceived stress. The PSS has good reliability and validity [2].

Pittsburgh sleep quality index (PSQI): The Pittsburgh Sleep Quality Index (PSQI) is an effective instrument used to measure the quality and patterns of sleep in adults. It differentiates "poor" from "good" sleep quality by measuring 
seven areas (components): subjective sleep quality, sleep latency, sleep duration, habitual sleep efficiency, sleep disturbances, use of sleeping medications, and daytime dysfunction over the last month. The PSQI has high test-retest reliability and a good validity [9].

\section{Data analysis}

Complete data was gathered in the form of a master chart made on Microsoft Excel 2010. The statistical analysis was conducted using Statistical Package for the Social Sciences 21 (SPSS v.21). Statistical significance at $\mathrm{p}</=0.05$ was assumed. A paired-samples t-test was conducted to analyse the effect of sleep hygiene program on sleep quality. Descriptive analysis included percentages, means and SD. Pearson correlation coefficient was calculated to find out the correlation between sleep and stress.

\section{Results}

A total of 70 elderly subjects participated in the study.

\section{Effectiveness of sleep hygiene program}

Sleep Hygiene Program found to be effective in improving the sleep quality of elderly $(\mathrm{M} \pm \mathrm{SD}=$ pre- $1.41 \pm .496$, post-2.64 \pm . 742, $\mathrm{t}=-9.772, \mathrm{p}=0.000)$ (Table 2).

Table 2. Effectiveness of sleep hygiene program on sleep quality.

\begin{tabular}{|c|c|c|c|c|c|}
\hline & & PRE & POST & & \\
\hline Variables & $\mathbf{N}$ & $M \pm S D$ & $M \pm S D$ & t-value & Significance level \\
\hline PSQI & 70 & $1.41 \pm .496$ & $2.64 \pm .742$ & $-9.772^{*}$ & 0.000 \\
\hline
\end{tabular}

*significant at 0.05 level; NS: not significant

\section{Stress level}

Within the study $14 \%(\mathrm{n}=10)$ participants had no stress, $31 \%$ $(n=22)$ had average stress level and 55\% $(n=38)$ participants had high stress level.

After the implementation of sleep hygiene program, stress level of the participants was reduced with $61 \%(n=43)$ had no stress, 26\% $(\mathrm{n}=18)$ participants had average stress level and $13 \%(n=9)$ participants had high stress level (Table 3$)$.

Table 3. Percentage analysis.

\begin{tabular}{|c|c|c|c|c|}
\hline \multicolumn{5}{|c|}{ Percentage Analysis } \\
\hline & Pre & & Post & \\
\hline & Frequency & Percentage & Frequency & Percentage \\
\hline \multicolumn{5}{|c|}{ PSQI SCORE (Sleep Quality) } \\
\hline Poor Sleep & 41 & $59 \%$ & 11 & $16 \%$ \\
\hline Very Poor Sleep & 29 & $41 \%$ & 3 & $4 \%$ \\
\hline Good Sleep & - & - & 56 & $80 \%$ \\
\hline
\end{tabular}

Perceived Stress Scale (PSS) Score

\begin{tabular}{lcccc}
\hline No stress & 10 & $14 \%$ & 43 & $61 \%$ \\
\hline Average & 22 & $31 \%$ & 18 & $26 \%$ \\
\hline High stress & 38 & $55 \%$ & 9 & $13 \%$ \\
\hline
\end{tabular}

\section{Correlation between sleep and stress}

Increase stress level was significantly associated with poor sleep quality with Pearson's $r$ values=pre- 0.95 , post- 0.92 (Table 4).

Table 4. Correlation between sleep and stress.

\begin{tabular}{lll}
\hline & \multicolumn{2}{l}{ Stress } \\
\cline { 2 - 3 } Pearson's r values & Pre & Post \\
\hline SLEEP & $0.95^{*}$ & $0.92^{*}$
\end{tabular}

* The Pearson's $r$ value is greater than 0 indicating a positive association between sleep and stress.

\section{Discussion}

This study was set out to analyse the effectiveness of sleep hygiene program on sleep quality and stress level among elderly population. Sample of 70 elderly subjects were taken from the community and they were analysed on the basis of different factors. Sleep quality and pattern was analysed using the Pittsburgh Sleep Quality Questionnaire. Participants having the PSQI score of 5 or above were taken in the study.

In the study, a sleep hygiene program was developed by the researcher to analyse its effect on sleep quality and stress level. SHP was provided to all the participants through face to face interview method for 4 days (over a period of 6 weeks) and then weekly follow-up was done through telephonic interview method. They were given a Sleep hygiene Booklet, for the follow up of sleep hygiene program at home. Within the study Sleep Hygiene Program found to be an effective tool in improving the sleep quality of elderly $(\mathrm{M} \pm \mathrm{SD}=$ pre- $1.41 \pm$ 0.496 , post- $2.64 \pm 0.742, \mathrm{t}=-9.772, \mathrm{p}=0.000$ )

Our result is supported by S. Blunden-et-al in 2012, who did a study to analyse whether sleep education change sleep parameters? They also compared sleep education trials for middle school students in Australia and New Zealand. They delivered the ACES program to 69 Australian adolescents and 29 New Zealand adolescents in a pre-post cross-sectional design. They concluded that ACES sleep education programmes can improve both sleep knowledge and sleep duration in adolescents [7].

The similar result was found by JinJu [8] in 2016, they did a study to investigate the effect of implementation of sleep hygiene education to elderly women people living in some local communities. In their study, through the result of impact of sleep hygiene program on sleep quality in the elderly women, Pittsburgh sleep disorder score in participation group was much lower than in non-participation $\operatorname{group}(\mathrm{t}=2.34$, $\mathrm{p}<0.05)$. Thus our results in line with these studies suggested that Sleep Hygiene Program is an important tool for improving the sleep quality of elderly. 
Citation: Sharma K, Srivastava S. The effectiveness of sleep hygiene program on sleep quality and stress level in elderly population. J Ment Health Aging 2018;2(1):29-32.

Cohen Perceived Stress Scale (PSS) was used to analyse the stress level and we identified that maximum number of participants had high stress level (55\%). The study also revealed that the stress level of the participants was reduced after the implementation of sleep hygiene program with $61 \%$ participants had no stress.

The findings showed that increase in stress level was significantly associated with poor sleep quality. Pearson correlation coefficient was calculated to find out the correlation between sleep and stress. Within the study it was found that stress and sleep was positively correlated, with Pearson's $r$ values $=$ pre- 0.95 , post- 0.92 .

Our result is supported by Kaur [2] in 2006, they did a Comparative study on psychosocial stresses among urban and rural geriatric population in selected areas of district Ludhiana (Punjab). Findings reveal that about $93 \%$ and $70 \%$ of subjects were in the mild psychosocial stress in urban and rural areas respectively while $30 \%$ of the rural subjects were in moderate psychosocial stress. Rural geriatric population was found more stressed than urban geriatric population and the most common stressful event in rural geriatric subjects was 'change in sleeping habits $(70 \%)$ [2]. Thus our results in line with these studies suggested that stress level is significantly associated with sleep quality.

Therefore, on the analysis of different factors it was clearly found that, the sleep hygiene program is an easily approachable and an effective intervention method for elderly population.

There were some limitations found during the study as both its small sample size and the age group. Moreover, its crosssectional design didn't permit us to completely evaluate the casual relationship. Future studies could focus on the larger sample size and participants of different age group, from different settings. Focus could also be given on one factor such as sleep pattern and quality, stress level [10-12].

\section{Conclusion}

Our study is one of the few available on the stress level and sleeps disorders faced by the elderly population. Within the study elderly population was found to be more stressed and the possible reason could be 'change in sleeping habits, problems in area of daily living and sudden loss of physical capabilities, occupations, friends, and spouse and independence. From the result we concluded that Sleep Hygiene Program is an important tool for improving the sleep quality and stress level of elderly. Within the study we found the positive association between sleep and stress.

Thus our study highlighted that poor sleep quality can lead to significantly greater psychosocial distress which had severe impact on elderly population and implementing the sleep hygiene program can be the best practice to improve the sleep quality and stress level in elderly population [8].

\section{References}

1. Sapkota A. Stress level among the geriatric population of urban area in eastern Nepal. Nepal Med Coll J. 2012;15(2):91-4.

2. Kaur M. Comparative study on psychosocial stresses among urban and rural geriatric population in selected areas of district Ludhiana (Punjab). Nurs Midwifery Res J. 2007;3(2).

3. Ali Ezzati MD. Validation of the perceived stress scale in a community sample of older adults. Int J Geriatr Psychiatry. 2014;29(6):645-52.

4. Suzuki K. Sleep disorders in the elderly: Diagnosis and management. J Gen Fam Med. 2017;18:61-71.

5. Khaje-Bishak Y. Assessing the quality of life in elderly people and related factors in Tabriz, Iran. J Caring Sci. 2014;3(4):257-63.

6. Stepnowsky CJ. Sleep and its disorders in seniors. Sleep Med Clin. 2008; 3(2):281-93.

7. Blunden S. Does sleep education change sleep parameters? Comparing Sleep Education Trials for Middle School Students in Australia and New Zealand. Open Sleep J. 2012;5:12-8.

8. JinJu S. The effects of sleep hygiene program on sleep quality in the elderly women. Indian J Sci Technol. 2016;9(37).

9. Brown FC. Relationship of sleep hygiene awareness, sleep hygiene practices and sleep quality in university students. Behav Med. 2002;28(1):33-8.

10. Daniel J. The pittsburgh sleep quality index as a new instrument for psychiatric practice and research. Psychiatr Res. 1988;28:193-213.

11. Monjan A. Perspective on sleep and aging. Front Neurol. 2010;1:124.

12. Wolkove N. Sleep and aging: Sleep disorders commonly found in older people. 2007;176(9):1299-304.

\section{*Correspondence to:}

Kiran Sharma

Department of Rehabilitation Sciences

Jamia Hamdard University

New Delhi

India

Tel: 07289994689

E-mail: kiran.somvati94@gmail.com 